\title{
Albert RAUGI \\ Dépassement des sommes partielles de v.a.r. indépendantes équidistribuées sans moment d'ordre 1
}

Annales de la faculté des sciences de Toulouse $6^{e}$ série, tome 9, no 4 (2000), p. 723-734

<http://www.numdam.org/item?id=AFST_2000_6_9_4_723_0>

(C) Université Paul Sabatier, 2000, tous droits réservés.

L'accès aux archives de la revue «Annales de la faculté des sciences de Toulouse » (http://picard.ups-tlse.fr/ annales/) implique l'accord avec les conditions générales d'utilisation (http://www.numdam.org/conditions). Toute utilisation commerciale ou impression systématique est constitutive d'une infraction pénale. Toute copie ou impression de ce fichier doit contenir la présente mention de copyright.

\section{Numdam}

Article numérisé dans le cadre du programme

Numérisation de documents anciens mathématiques

http://www.numdam.org/ 


\title{
Dépassement des sommes partielles de v.a.r. indépendantes équidistribuées sans moment d'ordre $1^{(*)}$
}

\author{
Albert RaUgi ${ }^{(1)}$
}

RÉSumÉ. - Soit $\left(X_{n}\right)_{n \geqslant 1}$ une suite de variables aléatoires réelles indépendantes positives de loi commune $\mu$. Nous étudions la nature de la série $\sum 1_{\left\{X_{n+1}>F\left(S_{n}\right)\right\}}$, pour une fonction strictement croissante et non bornée $F$ sur $\mathbb{R}_{+}$. En particulier, pour la fonction $F(x)=c x, c>0$, nous montrons que cette série est convergente ou divergente, $\mathbb{P}-$ p.s., selon que $\mu$ possède ou non un moment d'ordre 1 . Nous établissons aussi une "loi forte des grands nombres" pour certaines marches aléatoires sans moment d'ordre 1.

\begin{abstract}
Let $\left(X_{n}\right)_{n \geqslant 1}$ be a sequence of independant non negative real valued random variables with a common law $\mu$. Let $F$ be an increasing and unbounded function on $\mathbb{R}_{+}$. We study the convergence of the series $\sum 1_{\left\{X_{n+1}>F\left(S_{n}\right)\right\}}$. In particular, for the function $F(x)=c x, c>0$, we prove that this series converges $\mathbb{P}-$ a.s. if $\mathbb{E}\left[X_{1}\right]<+\infty$ and diverges $\mathbb{P}$-a.s. if $\mathbb{E}\left[X_{1}\right]=+\infty$. We also establish a "law of large numbers" for some random walks with undefined mean.
\end{abstract}

\section{Introduction}

Soit $\mu$ une mesure de probabilité sur les boréliens de $\mathbb{R}_{+}$, différente de la mesure de Dirac en zéro. Soit $\left(X_{n}\right)_{n \geqslant 1}$ une suite de variables aléatoires réelles indépendantes positives de loi commune $\mu$, définie sur un espace probabilisé

(*) Reçu le 26 avril 1999, accepté le 20 décembre 2000

(1) IRMAR, Université de Rennes 1, Campus de Beaulieu, 35042 Rennes cedex, France raugi@univ-rennes1.fr 
$(\Omega, \mathcal{F}, \mathbb{P})$. Pour tout entier $n \geqslant 1$, nous posons $S_{n}=X_{1}+\ldots+X_{n}$. Nous désignons par $H$ et $\Gamma$ les fonctions sur $\mathbb{R}_{+}$définies par :

$$
H(t)=\mu(] t,+\infty[) \quad \text { et } \quad \Gamma(t)=\int_{0}^{t} H(u) d u=\mathbb{E}\left[\min \left\{X_{1}, t\right\}\right] .
$$

Dans [1], il est montré que lorsque $H$ est de l'ordre de $\frac{1}{t}$ au voisinage de l'infini, alors les séries $\sum_{n \geqslant 1} 1_{\left\{X_{n+1}>S_{n}\right\}}$ et $\sum_{n \geqslant 1} H\left(S_{n}\right)$ divergent.

Nous considérons une fonction strictement croissante et non bornée $F$ sur $\mathbb{R}_{+}$et nous nous intéressons à la divergence presque sûre des séries

$$
\sum_{n \geqslant 1} 1_{\left\{X_{n+1}>F\left(S_{n}\right)\right\}}, \quad \sum_{n \geqslant 1} H \circ F\left(S_{n}\right) \text { et } \sum_{n \geqslant 1} \mathbb{E}\left[H \circ F\left(S_{n}\right)\right] .
$$

\section{Enoncés des résultats}

- Nature des séries (1).

(2.1) THÉORÈME. - Avec les notations précédentes, nous avons :

i) Les séries (1) sont de même nature.

ii) Si $\mathbb{E}\left[X_{1}\right]<+\infty$, les séries (1) convergent ou divergent, $\mathbb{P}-$ p.s., selon que l'intégrale $\int_{0}^{+\infty} H \circ F(t) d t$ est finie ou pas.

iii) Si $\mathbb{E}\left[X_{1}\right]=+\infty$, les séries (1) convergent ou divergent, $\mathbb{P}-$ p.s., selon que l'intégrale $\int_{1}^{+\infty} \frac{H \circ F(t)}{\Gamma(t)}\left(1-\frac{t H(t)}{\Gamma(t)}\right) d t$ est finie ou pas.

(2.2) Conollaine. - Pour tout réel $c>0$, les séries

$$
\sum_{n \geqslant 1} 1_{\left\{X_{n+1}>c S_{n}\right\}}, \quad \sum_{k \geqslant 0} H\left(c S_{n}\right) \text { et } \sum_{k \geqslant 0} \mathbb{E}\left[H\left(c S_{n}\right)\right]
$$

convergent ou divergent, $\mathbb{P}-$ p.s., selon que $\mathbb{E}\left[X_{1}\right]$ est finie ou pas.

\section{- Loi forte des grands nombres.}

(2.3) Hypothèse. - Nous introduisons la condition suivante :

$$
\text { il existe un réel } a \in] 0,1\left[\text { tel que } \int_{0}^{+\infty} \frac{t H^{2}(t)}{(\Gamma(t))^{2(1-a)}} d t<+\infty\right. \text {. }
$$


L'inégalité

$$
\int_{t}^{2 t} u H^{2}(u) \Gamma^{-2}(u) d u \geqslant H^{2}(2 t) \Gamma^{-2}(2 t) \int_{t}^{2 t} u d u \geqslant 3 / 2 t^{2} H^{2}(2 t) \Gamma^{-2}(2 t)
$$

montre que cette condition est plus forte que la condition

$$
\lim _{t \rightarrow+\infty} t H(t) / \Gamma(t)=0
$$

qui est une condition nécessaire et suffisante d'existence d'une loi faible des grands nombres pour le processus $\left(S_{n}\right)_{n \geqslant 1}$ (cf. [4], VII.7, p.236 ; [5]). La condition $\left(C_{2}\right)$ assure que la fonction $\Gamma$ est à variation lente au sens de Karamata (i.e. $\Gamma(r t) / \Gamma(t)$ tend vers 1 , pour tout $r>0$, quand $t$ tend vers $+\infty)$. Il suffit de remarquer que :

$$
\begin{aligned}
\Gamma(x)=\Gamma(1) \exp \left(\int_{1}^{x} \frac{H(t)}{\Gamma(t)} d t\right)=\Gamma(1) \exp \left(\int_{1}^{x} \frac{\varepsilon(t)}{t} d t\right) ; \\
\quad \text { où } \varepsilon(t)=t H(t) / \Gamma(t) .
\end{aligned}
$$

On notera aussi que, sous la condition $\left(C_{2}\right)$, l'intégrale de l'assertion iii) du théorème (2.1) peut être remplaçée par l'intégrale $\int_{1}^{+\infty} H \circ F(t) / \Gamma(t) d t$.

Pour $H(t) \underset{+\infty}{\sim} \exp \left(\int_{2}^{t} \frac{1}{u \log \log u} d u\right) / t \log \log t$ la condition $\left(C_{2}\right)$ est satisfaite alors que la condition $\left(C_{1}\right)$ ne l'est pas.

(2.4) THÉORÈmE. - Supposons que $\mathbb{E}\left[X_{1}\right]=+\infty$ et que la condition $\left(C_{1}\right)$ soit satisfaite. Pour tout $\alpha \in[0,1]$, appelons $\left(T_{n}(\alpha)\right)_{n \geqslant 1}$ le processus défini par :

$$
T_{n}(\alpha)=\sum_{k=1}^{n} \min \left\{X_{k}, n \Gamma^{1-\alpha}(n)\right\} .
$$

Alors : i) Pour tout $\alpha \in] 0,1]$, le processus $\left(T_{n}(\alpha) / n \Gamma(n)\right)_{n \geqslant 1}$ converge $\mathbb{P}$-presque sûrement vers 1 , quand $n$ tend vers $+\infty$.

ii) Pour tout $\alpha \in[0, a], T_{n}(\alpha) \leqslant S_{n} \leqslant \max \left\{X_{k}: 1 \leqslant k \leqslant n\right\}+T_{n}(\alpha)$.

\section{Démonstration des résultats}

- Démonstration des assertions $i$ ) et $i i)$ du théorème (2.1).

Le fait que les séries (1) soient de même nature découle des deux lemmes suivants. Le premier est une extension classique du lemme de Borel-Cantelli (cf [6], corollaire de la proposition IV-6-3). 
(3.1) LEMME. - Soit $\left(\mathcal{F}_{n}\right)_{n \geqslant 0}$ une suite croissante de sous-tribus de $\mathcal{F}$. Soit $\left(Z_{n}\right)_{n \geqslant 1}$ une suite de v.a.r., à valeurs dans $[0,1]$, adaptée à la filtration $\left(\mathcal{F}_{n}\right)_{n \geqslant 0}$ (i.e. pour tout entier $n \geqslant 1$, la v.a.r. $Z_{n}$ est $\mathcal{F}_{n}$-mesurable).

Alors

$$
\left\{\sum_{n \geqslant 1} Z_{n}<+\infty\right\}=\left\{\sum_{n \geqslant 1} \mathbb{E}\left[Z_{n} \mid \mathcal{F}_{n-1}\right]<+\infty\right\}, \mathbb{P}-\text { p.s. }
$$

(3.2) Lemme. - Considérons l'espace produit $\Omega=\mathbb{R}^{\mathbb{N}^{*}}$ muni de la probabilité produit $\mathbb{P}=\otimes_{\mathbb{N}^{*}}^{\otimes} \mu$. Nous désignons par $\left(X_{n}\right)_{n \geqslant 1}$ les applications coordonnées de $\Omega$ et par $\theta$ l'opérateur de décalage sur $\Omega$ (i.e. $\forall n \geqslant 1, X_{n}$ 。 $\left.\theta=X_{n+1}\right)$. Pour tout entier $n \geqslant 1$, nous notons $\mathcal{F}_{n}$ la tribu engendrée par les v.a.r. $X_{k}, 1 \leqslant k \leqslant n$. Soit $\left(Z_{n}\right)_{n \geqslant 1}$ une suite de v.a.r. à valeurs dans $[0,1]$, adaptée à la filtration $\left(\mathcal{F}_{n}\right)_{n \geqslant 0}$ et vérifiant $\forall n \geqslant 1, Z_{n} \circ \theta \geqslant Z_{n+1}$.

Si $\sum_{k=1}^{n} \mathbb{E}\left[Z_{k}\right]=+\infty$ alors $\limsup _{n \rightarrow+\infty}\left(\sum_{k=1}^{n} Z_{k} / \sum_{k=1}^{n} \mathbb{E}\left[Z_{k}\right]\right) \geqslant 1, \mathbb{P}$-p.s..

Preuve. - Pour tout entier $n \geqslant 1$, posons $F_{n}=\sum_{k=1}^{n} Z_{k} / \sum_{k=1}^{n} \mathbb{E}\left[Z_{k}\right]$. La v.a.r. $F=\limsup _{n \rightarrow+\infty} F_{n}$ est $\theta$-sous-invariante (i.e. $F \circ \theta \geqslant F$ ) et par suite $\theta$-presque sûrement invariante (i. e. $F \circ \theta=F, \mathbb{P}$-p.s.). Noter que, pour tout réel positif $a$, la v.a.r. $\mathbb{P}$-intégrable $F_{a}=F \wedge a$ est $\theta$-sous-invariante et vérifie $\mathbb{E}\left[F_{a} \circ \theta\right]=\mathbb{E}\left[F_{a}\right]$ grâce à l'invariance de $\mathbb{P}$ par $\theta$. D'après la loi 0-1 de Kolmogorov, la v.a.r. $F$ est donc $\mathbb{P}$-p.s. constante.

Il suffit alors de montrer que la suite de v.a.r. $\left(F_{n}\right)_{n \geqslant 1}$ est équi-intégrable ; en effet dans ce cas on sait que l'on a l'inégalité de Fatou-Lebesgue

$$
\mathbb{E}\left[\limsup _{n \rightarrow+\infty} F_{n}\right] \geqslant \limsup _{n \rightarrow+\infty} \mathbb{E}\left[F_{n}\right]=1 .
$$

Pour cela nous montrons que $\sup _{n \geqslant 1} \mathbb{E}\left[F_{n}^{2}\right]<+\infty$. Pour tous entiers $\ell$ et $k$ supérieurs ou égaux à 1 , nous avons :

$$
\mathbb{E}\left[Z_{\ell} Z_{\ell+k}\right] \leqslant \mathbb{E}\left[Z_{\ell} Z_{k} \circ \theta^{\ell}\right]=\mathbb{E}\left[Z_{\ell}\right] \mathbb{E}\left[Z_{k}\right]
$$

et par suite

$$
\mathbb{E}\left[\left(\sum_{k=1}^{n} Z_{k}\right)^{2}\right] \leqslant \sum_{k=1}^{n} \mathbb{E}\left[Z_{k}\right]+2 \sum_{1 \leqslant \ell \leqslant k \leqslant n} \mathbb{E}\left[Z_{\ell}\right] \mathbb{E}\left[Z_{k-\ell}\right] \leqslant 3\left(\sum_{k=1}^{n} \mathbb{E}\left[Z_{k}\right]\right)^{2},
$$

pour $n$ assez grand pour que $\sum_{k=1}^{n} \mathbb{E}\left[Z_{k}\right] \geqslant 1$. D'où le résultat.

Lorsque $\mathbb{E}\left[X_{1}\right]<+\infty$, il résulte de la loi forte des grands nombres et de la décroissance de la fonction $H \circ F$ que : pour tout $\varepsilon>0$, pour $\mathbb{P}$-presque 
tout $\omega \in \Omega$, il existe un entier $N(\omega)$ tel que pour tout entier $n \geqslant N(\omega)$,

$$
H \circ F\left(n\left(\mathbb{E}\left[X_{1}\right]+\varepsilon\right)\right) \leqslant H \circ F\left(S_{n}(\omega)\right) \leqslant H \circ F\left(n\left(\mathbb{E}\left[X_{1}\right]-\varepsilon\right)\right) .
$$

Or, pour tout réel $r>0$, la série $\sum_{n \geqslant 1} H \circ F(r n)$ est de même nature que l'intégrale $\int_{0}^{+\infty} H \circ F(t) d t$. D'où la seconde assertion du théorème (2.1).

\section{- Préparatifs au cas $\mathbb{E}\left[X_{1}\right]=+\infty$.}

(3.3). - Dorénavant nous supposons que $\mathbb{E}\left[X_{1}\right]=+\infty$. Nous considérons la fonction $\varphi$ définie sur $\mathbb{R}_{+}^{*}$ par :

$$
\varphi(x)=\frac{x}{\Gamma(x)}=\frac{t}{\mathbb{E}\left[\min \left\{X_{1}, x\right\}\right]}=\frac{1}{\mathbb{E}\left[\min \left\{X_{1} / x, 1\right\}\right]} .
$$

La dernière égalité montre que : la fonction $\varphi$ peut être prolongée par continuité en zéro par $\varphi(0)=1$ et $\lim _{x \rightarrow+\infty} \varphi(x)=+\infty$.

La fonction $H$ est positive, décroissante et continue à droite sur $\mathbb{R}_{+}^{*}$. La fonction $\Gamma$ est croissante, continue, dérivable à droite et à gauche, avec

$$
\forall x \in] 0,+\infty\left[, \quad \Gamma_{d}^{\prime}(x)=H(x) \quad \text { et } \quad \Gamma_{g}^{\prime}(x)=H(x-0) .\right.
$$

L'ensemble $\left\{x \in \mathbb{R}_{+}: H(x-0)<H(x)\right\}$ est dénombrable. La fonction $\Gamma$ est donc Lebesgue-presque partout dérivable. De plus, $\lim _{x \rightarrow+\infty} \Gamma(x)=\mathbb{E}[X]=+\infty$. La fonction $\varphi$ est continue, dérivable à droite et à gauche sur $\mathbb{R}$, et

$$
\forall x \in] 0,+\infty\left[, \quad \varphi_{d}^{\prime}(x)=\frac{\Gamma(x)-x H(x)}{\Gamma^{2}(x)}=\frac{\mathbb{E}\left[X_{1} 1_{\left\{X_{1} \leqslant x\right\}}\right]}{\Gamma^{2}(x)} .\right.
$$

On en déduit donc que la fonction $\varphi$ est croissante et même strictement croissante sur un intervalle $[s,+\infty[, s>0$. De plus $\forall x \geqslant 0, \varphi(x)=\varphi(0)+$ $\int_{0}^{x} \varphi_{d}^{\prime}(t) d t$.

\section{- Démonstration de l'assertion iii) du théorème (2.1) .}

L'assertion iii) du théorème (2.1) résulte de l'encadrement classique suivant concernant la mesure "potentiel" (cf. [3], lemme 1).

(3.4) Lemme. - Pour tout entier $k \geqslant 1$, nous notons $\mu^{k *}$ la $k$-ième convolée de $\mu$ et nous appelons $G$ la mesure "potentiel" $\sum_{k \geqslant 0} \mu^{k *}$, où $\mu^{0}$ est la mesure de Dirac $\delta_{0}$ en zéro. 
Alors, pour tout réel strictement positif $t, \varphi(t) \leqslant G([0, t]) \leqslant 2 \varphi(t)$.

Preuve. - De la relation $\left(\delta_{0}-\mu\right) * G=\delta_{0}$, il s'ensuit que

$$
\forall x \geqslant 0, \int_{0}^{x}\left(\left(\delta_{0}-\mu\right) * G\right)([0, t]) d t=\int_{0}^{x} \delta_{0}([0, t]) d t ;
$$

c'est-à-dire $\forall x \geqslant 0, \int_{0}^{x} \int_{0}^{+\infty} 1_{[0, t]}(u) H(t-u) d G(u) d t=x$. Par application du théorème de Fubini et changement de variable $v=t-u$, il vient, pour tout $x \geqslant 0$,

$x=\int_{0}^{+\infty} 1_{[0, x]}(u)\left(\int_{u}^{x} H(t-u) d t\right) d G(u)=\int_{0}^{+\infty} 1_{[0, x]}(u) \Gamma(x-u) d G(u)$.

De la croissance de la fonction $\Gamma$, il résulte alors que, pour tout $x \geqslant 0$,

$$
\Gamma(x / 2) G([0, x / 2]) \leqslant \int_{0}^{+\infty} 1_{[0, x / 2]}(u) \Gamma(x-u) d G(u) \leqslant x \leqslant \Gamma(x) G([0, x]) .
$$

D'où le résultat.

En posant $S_{0}=0$, nous avons, via le théorème de Fubini,

$$
\sum_{n \geqslant 0} \mathbb{E}\left[H \circ F\left(S_{n}\right)\right]=\int_{0}^{+\infty} H \circ F(t) d G(t)=\int_{0}^{+\infty} G\left(\left[0, F^{-1}(u)[) d \mu(u)\right.\right.
$$

et par suite, en tenant compte du lemme (3.4),

$$
\int_{0}^{+\infty} \varphi\left(F^{-1}(u) / 2\right) d \mu(u) \leqslant \sum_{n \geqslant 0} \mathbb{E}\left[H \circ F\left(S_{n}\right)\right] \leqslant 2 \int_{0}^{+\infty} \varphi\left(F^{-1}(u)\right) d \mu(u) .
$$

Or $\quad \varphi(x / 2)=\varphi(x)(\Gamma(x) / 2 \Gamma(x / 2)) \geqslant \varphi(x)(\Gamma(x / 2) / 2 \Gamma(x / 2))=\varphi(x) / 2$

et

$$
\begin{aligned}
\int_{0}^{+\infty} \varphi\left(F^{-1}(u)\right) d \mu(u) & =\varphi(0)+\int_{0}^{+\infty} \int_{0}^{F^{-1}(u)} \varphi_{d}^{\prime}(y) d y d \mu(u) \\
& =\varphi(0)+\int_{0}^{+\infty} H \circ F(y) \varphi_{d}^{\prime}(y) d y
\end{aligned}
$$

D'où l'assertion iii) du théorème (2.1). 


\section{- Démonstration du corollaire (2.2) .}

Nous venons de montrer que les séries (1) convergent, $\mathbb{P}$-p.s., si et seulement si $\mathbb{E}\left[\varphi\left(F^{-1}\left(X_{1}\right)\right)\right]<+\infty$. Pour $F(t)=c t$ avec $c>0$, les séries (1) convergent si et seulement si $\mathbb{E}\left[\varphi\left(c^{-1} X_{1}\right)\right]<+\infty$. Le corollaire résulte alors du lemme suivant.

(3.5) Lemme. - Soit $X$ une variable aléatoire réelle. Si $\mathbb{E}[X]=+\infty$, alors $\mathbb{E}[\varphi(X)]=+\infty$.

Preuve. - Nous avons :

$\mathbb{E}[\varphi(X)]-\varphi(0)=\int_{0}^{+\infty} H(u) \varphi_{d}^{\prime}(t) d t=\int_{0}^{+\infty} H(t) \Gamma^{-1}(t)\left(1-t H(t) \Gamma^{-1}(t)\right) d t$.

Si la mesure de probabilité $\mu$ vérifie l'hypothèse $\left(C_{2}\right)$, la dernière intégrale est de même nature que l'intégrale $\int_{1}^{+\infty} H(t) \Gamma^{-1}(t) d t$ qui diverge. Si $\mu$ ne vérifie pas la condition $\left(C_{2}\right)$, alors l'inégalité

$$
\forall t>0, \mathbb{E}\left[\varphi(X) 1_{\{X>t\}}\right] \geqslant H(t) \varphi(t)=t H(t) \Gamma^{-1}(t)
$$

montre que nécessairement $\mathbb{E}[\varphi(X)]=+\infty$.

\section{- Démonstration du théorème (2.4) .}

Cette démonstration résultera de trois lemmes.

Pour tout $\alpha \in[0,1]$, nous posons :

$$
\varphi_{\alpha}(t)=t^{\alpha}(\varphi(t))^{1-\alpha} .
$$

Les fonctions $\varphi_{\alpha}$ sont strictement croissantes sur un intervalle $[s,+\infty[, s>$ 0 . Nous notons $\beta_{\alpha}$ la fonction réciproque de $\varphi_{\alpha}$ et nous supposons que $s$ est assez grand pour que $\Gamma(s)>1$. Pour tout $t \geqslant s, \varphi(t) \leqslant t$; il s'ensuit que $\varphi_{\alpha}(t)$ croît en $\alpha$ et $\beta_{\alpha}(t)$ décroît en $\alpha$. Pour alléger l'écriture, nous notons $\beta$ la fonction $\beta_{0}$.

(3.6) Lemme. - Nous avons :

i) Pour tout $\alpha \in[0,1]$ et tout réel $t>s, \beta_{\alpha}(t)=t\left(\Gamma\left(\beta_{\alpha}(t)\right)\right)^{1-\alpha}$.

ii) Sous l'hypothèse $\left(C_{1}\right)$ de (2.3), pour tout $\alpha \in[0,1], \Gamma\left(\beta_{\alpha}(t)\right) \underset{+\infty}{\sim} \Gamma(t)$.

iii) Appelons $G_{\alpha}$ la fonction $x \rightarrow x^{-2} \Gamma^{-2}(x) \int_{\beta_{\alpha}(s)}^{\beta_{\alpha}(x)} u H(u) d u$. La fonction $G_{1}$ est intégrable sur $\left[s,+\infty\left[\right.\right.$. Sous l'hypothèse $\left(C_{1}\right)$, la fonction $G_{\alpha}$ est intégrable sur $[s,+\infty[$, pour tout $\alpha \in] 0,1]$. 
iv) Sous l'hypothèse $\left(C_{1}\right)$, pour tout $r>0$, la fonction $t H^{2}\left(r \beta_{a}(t)\right)$ est intégrable sur $[s,+\infty[$.

Preuve. - La première assertion est évidente.

Des diverses monotonies soulignées auparavant, il résulte que

$$
1 \leqslant \Gamma\left(\beta_{\alpha}(t)\right) / \Gamma(t) \leqslant \Gamma(\beta(t)) / \Gamma(t) .
$$

Il suffit donc de montrer l'assertion $i i$ ), pour $\alpha=0$. Nous avons

$$
\begin{aligned}
\log \frac{\Gamma(\beta(t))}{\Gamma(t)} & =\int_{t}^{\beta(t)} \frac{H(u)}{\Gamma(u)} d u \\
& \leqslant \sqrt{\int_{t}^{\beta(t)} \frac{u H^{2}(u)}{\Gamma^{2(1-a)}(u)} d u} \sqrt{\int_{t}^{\beta(t)} \frac{\Gamma^{-2 a}(u)}{u} d u} \\
& \left.\leqslant C \Gamma^{-a}(t) \sqrt{\log \frac{\beta(t)}{t}} \text { (hypothèse }\left(C_{2}\right)\right) \\
& \leqslant C \Gamma^{-a}(t) \sqrt{\log \Gamma(\beta(t))} \\
& \leqslant C \Gamma^{-a}(t) \sqrt{\log \frac{\Gamma(\beta(t))}{\Gamma(t)}+\log \Gamma(t) .}
\end{aligned}
$$

Considérons la fonction $g(t)=\log \frac{\Gamma(\beta(t))}{\Gamma(t)}$. Soit $\left(t_{n}\right)_{n \geqslant 0}$ une suite réelle tendant vers $+\infty$ tels que $\lim _{n \rightarrow+\infty} g\left(t_{n}\right)=\lambda \in[0,+\infty]$ et $g\left(t_{n}\right)>0$, pour tout $n \geqslant 0$. L'inégalité précédente, nous donne

$$
\forall n \geqslant 0, \sqrt{g\left(t_{n}\right)} \leqslant C \sqrt{\frac{1}{\Gamma^{2 a}\left(t_{n}\right)}+\frac{\log \Gamma\left(t_{n}\right)}{\Gamma^{2 a}\left(t_{n}\right) g\left(t_{n}\right)}}
$$

qui implique $\lambda=0$. On en déduit que $\lim _{t \rightarrow+\infty} g(t)=0$. D'où l'assertion $\left.i i\right)$.

Nous montrons la seconde affirmation de $i i i)$; la première se démontrant de la même façon. D'après les assertions $i$ ) et $i i), \Gamma(t) \sim \Gamma\left(\beta_{\alpha}(t)\right)$. Nous sommes donc amenés à prouver l'intégrabilité de la fonction

$$
L(t)=t^{-2} \Gamma^{-2}\left(\beta_{\alpha}(t)\right) \int_{\beta_{\alpha}(s)}^{\beta_{\alpha}(t)} u H(u) d u .
$$

Notons tout d'abord que l'égalité de définition de $\varphi_{\alpha}$ nous donne, en prenant les dérivées logarithmiques :

$$
\frac{\varphi_{\alpha}^{\prime}(t)}{\varphi_{\alpha}(t)}=\frac{\alpha}{t}+(1-\alpha) \frac{\varphi^{\prime}(t)}{\varphi(t)} \leqslant \frac{\alpha}{t}+(1-\alpha) \frac{H(t)}{t} \leqslant 1 / t .
$$


Dépassement des sommes partielles de v.a.r.

Nous avons alors :

$$
\begin{aligned}
\int_{s}^{+\infty} L(t) d t & =\int_{\beta_{\alpha}(s)}^{+\infty} \frac{\int_{\beta_{\alpha}(s)}^{t} u H(u) d u}{\Gamma^{2}(t) \varphi_{\alpha}^{2}(t)} \varphi_{\alpha}^{\prime}(t) d t \\
& \leqslant \int_{\beta_{\alpha}(s)}^{+\infty} \frac{\int_{\beta_{\alpha}(s)}^{t} u H(u) d u}{t^{2} \Gamma^{1+\alpha}(t)} d t \\
& \leqslant \int_{\beta_{\alpha}(s)}^{+\infty} u H(u) \int_{u}^{+\infty} \frac{d t}{t^{2} \Gamma^{1+\alpha}(t)} d u \\
& \leqslant \int_{\beta_{\alpha}(s)}^{+\infty} \frac{u H(u)}{\Gamma^{1+\alpha}(u)} \int_{u}^{+\infty} \frac{d t}{t^{2}} d u \\
& \leqslant \int_{\beta_{\alpha}(s)}^{+\infty} \frac{H(u)}{\Gamma^{1+\alpha}(u)} d u=\frac{1}{\alpha} \Gamma^{-\alpha}\left(\beta_{\alpha}(s)\right)<+\infty .
\end{aligned}
$$

L'assertion $i v$ ) résulte de :

$$
\int_{s}^{+\infty} u H^{2}\left(r \beta_{a}(u)\right) d u \leqslant \int_{\beta_{a}(s)}^{+\infty} \frac{u H^{2}(r u)}{\Gamma^{2-2 a}(u)} d u<+\infty
$$

Pour tout $\alpha \in[0,1]$ et tout réel $t \geqslant s$, posons :

$$
T_{t}(\alpha)=\sum_{k=1}^{[t]} X_{k} \wedge \beta_{\alpha}(t)+\{t\} X_{[t]+1} \wedge \beta_{\alpha}(t) ;
$$

où $[t]$ et $\{t\}$ désigne respectivement les parties entière et fractionnaire de $t$. Alors nous avons le résultat suivant :

(3.7) Lemme. - Supposons que $\mathbb{E}\left[X_{1}\right]=+\infty$. Si la condition $\left(C_{1}\right)$ est satisfaite, alors pour tout $\alpha \in] 0,1]$, le processus $\left(T_{t}(\alpha) / t \Gamma(t)\right)_{t \geqslant s}$ converge $\mathbb{P}$-p.s. vers 1 , quand $t$ tend vers $+\infty$. Lorsque seulement la condition $\left(C_{2}\right)$ est satisfaite, cette convergence a lieu pour $\alpha=1$.

Preuve. - Pour $t \geqslant s$, nous avons :

$$
E\left[T_{t}\right]=t \mathbb{E}\left[X_{1} \wedge \beta_{\alpha}(t)\right]=t \Gamma\left(\beta_{\alpha}(t)\right) .
$$

Pour $\alpha=1, E\left[T_{t}\right]=t \Gamma(t)$. Pour $\alpha \in\left[0,1\left[, \mathbb{E}\left[T_{t}\right] \underset{+\infty}{\sim} t \Gamma(t)\right.\right.$, d'après les assertions $i$ ) et $i i)$ du lemme (3.6).

De même, nous avons :

$$
\operatorname{var}\left(T_{t}\right)=t \operatorname{var}\left(X_{1} \wedge \beta_{\alpha}(t)\right) \leqslant t \mathbb{E}\left[X_{1}^{2} \wedge \beta_{\alpha}^{2}(t)\right]=t \int_{0}^{\beta_{\alpha}(t)} 2 u H(u) d u .
$$


D'où la majoration,

$$
\operatorname{var}\left(\frac{T_{t}}{t \Gamma(t)}\right) \leqslant 2 t \frac{\int_{0}^{\beta_{\alpha}(t)} u H(u) d u}{t^{2} \Gamma^{2}(t)} .
$$

Pour tout réel $r>1$, nous avons alors :

$$
\begin{aligned}
\operatorname{var}\left(\frac{T_{r^{n}}}{r^{n} \Gamma\left(r^{n}\right)}\right) & \leqslant 2 r^{n} \frac{\int_{0}^{\beta_{\alpha}\left(r^{n}\right)} u H(u) d u}{r^{2 n} \Gamma^{2}\left(r^{n}\right)} \\
& \leqslant \frac{2}{r-1} \frac{r^{2(n+1)} \Gamma^{2}\left(r^{n+1}\right)}{r^{2 n} \Gamma^{2}\left(r^{n}\right)} \int_{r^{n}}^{r^{n+1}} \frac{\int_{0}^{\beta_{\alpha}(t)} u H(u) d u}{t^{2} \Gamma^{2}(t)} d t \\
& \leqslant \frac{2 r^{2}}{r-1} \frac{\Gamma^{2}\left(r^{n+1}\right)}{\Gamma^{2}\left(r^{n}\right)} \int_{r^{n}}^{r^{n+1}} \frac{\int_{0}^{\beta_{\alpha}(t)} u H(u) d u}{t^{2} \Gamma^{2}(t)} d t
\end{aligned}
$$

Lorsque la condition $\left(C_{2}\right)$ est satisfaite, $\Gamma$ est une fonction à variation lente. De l'assertion $i i i)$ du lemme (3.6), il résulte alors que la série $\sum \operatorname{var}\left(\frac{T_{r n}}{r^{n} \Gamma\left(r^{n}\right)}\right)$ converge pour tout $r>1$. D'où l'on déduit la convergence $\mathbb{P}-$ p.s. vers $1 \mathrm{de}$ la suite de v.a.r. $\left.\left(T_{r^{n}} / r^{n} \Gamma\left(r^{n}\right)\right)\right)_{n \geqslant 1}$, pour tout $r>1$.

Pour tout réel $t \geqslant s$, considérons l'entier $n=\left[\frac{\log t}{\log r}\right]$. Nous avons $r^{n} \leqslant$ $t \leqslant r^{n+1}$ et par suite

$$
\frac{T\left(r^{n}\right)}{r^{n+1} \Gamma\left(r^{n+1}\right)} \leqslant \frac{T(t)}{t \Gamma(t)} \leqslant \frac{T\left(r^{n+1}\right)}{r^{n} \Gamma\left(r^{n}\right)} .
$$

Comme $\Gamma$ est une fonction à variation lente, la convergence précédente montre que, $\mathbb{P}$-p.s.,

$$
\frac{1}{r} \leqslant \liminf _{t \rightarrow+\infty} \frac{T(t)}{t \Gamma(t)} \leqslant \limsup _{t \rightarrow+\infty} \frac{T(t)}{t \Gamma(t)} \leqslant r .
$$

On obtient le résultat voulu, en faisant tendre $r$ vers 1 .

Le lemme suivant est inspiré du lemme 2 de [2].

(3.8) Lemme. - Plaçons nous sous les hypothèses du théorème (2.4). Soit $\alpha \in[0, a]$. Pour $\mathbb{P}$-presque tout $\omega \in \Omega$, il existe un entier $N(\omega)$ tel que, pour tout entier $n \geqslant N(\omega)$, il existe au plus un entier $k$ de $\{1, \ldots, n\}$ pour lequel $X_{k}>\beta_{\alpha}(n)$.

Preuve. - Soit $r \in] 0,1[$. Pour tout entier $p \geqslant 1$, posons :

$$
A_{p}=\bigcup_{1 \leqslant \ell<k \leqslant p}\left\{X_{\ell}>r \beta_{\alpha}(p)\right\} \cap\left\{X_{k}>r \beta_{\alpha}(p)\right\} .
$$


Nous avons

$$
\mathbb{P}\left[A_{p}\right] \leqslant \sum_{1 \leqslant \ell<k \leqslant p} H^{2}\left(r \beta_{\alpha}(p)\right) \leqslant p^{2} H^{2}\left(r \beta_{\alpha}(p)\right) .
$$

Or, pour tout entier $m$, nous avons

$$
2^{2 m} H^{2}\left(r \beta_{\alpha}\left(2^{m}\right)\right) \leqslant 2^{2} \int_{2^{m-1}}^{2^{m}} t H^{2}\left(r \beta_{\alpha}(t)\right) d t .
$$

D'après l'assertion $i v$ ) du Lemme (3.6), la série $\sum_{m \geqslant 0} \mathbb{P}\left[A_{2^{m}}\right]$ est convergente et, pour $\mathbb{P}$-presque tout $\omega$, il existe un entier $N_{1}(\omega) \geqslant \log _{2} s$ tel que, pour tout entier $m \geqslant N_{1}(\omega)$, il existe au plus un entier $k$ de $\left\{1, \ldots, 2^{m}\right\}$ pour lequel $X_{k} \geqslant r \beta_{\alpha}\left(2^{m}\right)$. Il s'ensuit que pour tout entier $n>2^{N_{1}(\omega)}$, il existe au plus un entier $k$ de $\left\{1, \ldots, 2^{\left[\log _{2} n\right]+1}\right\}$ pour lequel $X_{k} \geqslant r \beta_{\alpha}\left(2^{\left[\log _{2} n\right]+1}\right)$. Ce qui implique qu'il existe au plus un entier $k$ de $\{1, \ldots, n\}$ tel que $X_{k} \geqslant r^{\prime} \beta_{\alpha}(n)$, avec $r^{\prime}=r \sup _{p \geqslant s} \beta(2 p) / \beta(p)$. On notera que, comme $\beta(t) \sim t \Gamma(t)$ et $\Gamma$ est à variation lente, la quantité $\sup _{p \geqslant s} \beta(2 p) / \beta(p)$ est finie. On obtient le résultat voulu, en choisissant $r$ de façon que $r^{\prime} \leqslant 1$.

L'assertion $i$ ) du théorème (2.4) résulte des lemmes (3.6) et (3.7), en notant que, pour tous réels $\alpha_{1}, \alpha, \alpha_{2}$ vérifiant $0 \leqslant \alpha_{1} \leqslant \alpha \leqslant \alpha_{2} \leqslant 1$,

$$
X_{1} \wedge \beta_{\alpha_{2}}(t) \leqslant X_{1} \wedge t \Gamma^{1-\alpha}(t) \leqslant X_{1} \wedge \beta_{\alpha_{1}}(t),
$$

pour $t$ suffisamment grand. L'assertion $i i$ ) résulte du lemme (3.8).

(3.9) Remarques.

1) Si la mesure de probabilité $\mu$ vérifie l'hypothèse $\left(C_{2}\right)$, alors on vérifie facilement que la mesure de probabilité $\varphi(\mu)$ vérifie l'hypothèse $\left(C_{1}\right)$.

2) Sous l'hypothèse $\left(C_{1}\right)$, on peut obtenir l'assertion $\left.i i i\right)$ du théorème (2.1) à partir du théorème (2.4). Soit $\alpha \in] 0, a]$. D'après les assertions $i$ ) et ii), pour $\mathbb{P}$-presque tout $\omega \in \Omega$, il existe un entier $N(\omega)$ tel que, pour tout $n \geqslant N(\omega)$,

$$
\frac{1}{2} \beta(n) \leqslant S_{n}(\omega) \leqslant \max \left\{X_{k}(\omega): 1 \leqslant k \leqslant n\right\}+2 \beta(n) .
$$

On voit facilement que, pour tout $r>0$, la série $\sum_{n \geqslant 1} H \circ F(r \beta(n))$ est de même nature que l'intégrale $\int_{1}^{+\infty} H \circ F(t) / \Gamma(t) d t$. Lorsque cette intégrale converge, de la décroissance de $H \circ F$, il résulte que la série $\sum_{n \geqslant 1} H \circ F\left(S_{n}\right)$ converge, $\mathbb{P}$-presque sûrement. Lorsque l'intégrale diverge, nous montrons la 
divergence, $\mathbb{P}$-p.s., de la série $\sum_{n \geqslant 1} H \circ F\left(M_{n}+2 \beta(n)\right)$, où $M_{n}=\max \left\{X_{k}\right.$ : $1 \leqslant k \leqslant n\}$.

Pour tout entier $n$, nous avons :

$$
\sum_{k=1}^{n} H \circ F\left(M_{k}+2 \beta(k)\right) \geqslant \sum_{k=1}^{n} H \circ F(4 \beta(k)) 1_{\left\{M_{k} \leqslant 2 \beta(k)\right\}}
$$

Appelons $Z$ la v.a.r. positive $\sum_{k \geqslant 1} H \circ F(4 \beta(k)) 1_{\left\{M_{k} \leqslant 2 \beta(k)\right\}}$. Pour tout entier naturel $p$, nous avons :

$$
\mathbb{E}\left[Z 1_{\{Z \leqslant p\}}\right]=\sum_{k \geqslant 1} H \circ F(4 \beta(k)) \mathbb{P}\left[\left\{M_{k} \leqslant 2 \beta(k)\right\} \cap\{Z \leqslant p\}\right] .
$$

Or

$$
\mathbb{P}\left[\left\{M_{k} \leqslant 2 \beta(k)\right\}\right]=(1-H(2 \beta(k)))^{k} \geqslant 1-k H(2 \beta(k))
$$

et $k H(2 \beta(k))=\beta(k) H(2 \beta(k)) / \Gamma(\beta(k))$ tend vers zéro quand $k$ tend vers l'infini, car $\Gamma$ est à variation lente et la condition $C_{2}$ est satisfaite. On en déduit que $\mathbb{P}\left[\left\{M_{k} \leqslant 2 \beta(k)\right\} \cap\{Z \leqslant p\}\right]$ tend vers $\mathbb{P}[\{Z \leqslant p\}]$ quand $k$ tend vers l'infini. Comme la série $\sum_{k \geqslant 1} H \circ F(4 \beta(k))$ diverge, nous avons nécessairement $\mathbb{P}[\{Z \leqslant p\}]=0$, pour tout entier naturel $p$. D'où le résultat.

\section{Bibliographie}

[1] Conze (J.-P.), Guivarc'H (Y.), Raugi (A.). - Dépassement des sommes partielles pour des v.a. sans moment, Séminaire de Rennes, 1998.

[2] Diamond (H.-G.), VAaler (J.-D.). - Estimates for partial sums of continued fraction partial quotients, Pacific J. of Math, Vol. 122, No 1, 1986.

[3] ERICKSON (K.-B.). - The strong law of large number when the mean is undefined, Trans. Am. Math. Soc., vol. 185, 1973, p. 371-381.

[4] Feller (W.). - An introduction to probability Theory and its applications, vol II, John Wiley \& Sons, 1971.

[5] Kesten (H.), Maller (R.-A.). - Ratios of trimmed sums and order statistis, Ann. Probab., 20, 1992, p. 1805-1842.

[6] Neveu (J.). - Bases mathématiques du calcul des probabilités, Masson, 1970. 